\title{
Identification of Anaerobic Threshold during Dynamic Exercise in Healthy Men Using Kolmogorov-Sinai Entropy
}

\author{
FMHSP Silva $^{1}$, AC Silva Filho ${ }^{2}$, LO Murta $\mathrm{Jr}^{1}$, MAS Lavrador ${ }^{1}$, VRFS Marães ${ }^{3}$, MS Moura ${ }^{3}$, \\ AM Catai $^{3}$, E Silva $^{3}$, BC Maciel $^{1}$, L Gallo Jr $^{1}$ \\ ${ }^{1}$ University of São Paulo, Ribeirão Preto, Brazil \\ ${ }^{2}$ University of Ribeirão Preto, Ribeirão Preto, Brasil \\ ${ }^{3}$ Federal University of São Carlos, São Carlos, Brazil
}

\begin{abstract}
During dynamic physical exercise there is a changing point in physiological state called Anaerobic Threshold $(A T)$. Some respiratory and cardiovascular variables, including heart rate variability $(H R V)$, experiment substantial changes at this point. In this work we measure the AT using Kolmogorov-Sinai Entropy applied to HRV time series. This procedure has two major advantage: a) it is non-invasive and b) it requests low cost equipments. The study also includes the comparison of AT values obtained by the mentioned method with another one obtained through a statistical analysis using the Auto Regressive Integrated Moving Average model.
\end{abstract}

\section{Introduction}

During dynamic physical exercise (DE) there is a changing point in physiological state called Anaerobic Threshold (AT) [1]. Changes in cardio respiratory variables including heart rate variability occur at this point $[1,2]$. The search for low cost, non-invasive methods for AT identification, has raised interests from researchers that work in the signal processing applied to biological systems field. Auto Regressive Integrated Moving Average model (ARIMA) has been used for this purpose [3-5].

The general idea to be understood in the entropy concept is that it is impossible to use all the system energy involved in a work realization, because part of that energy is lost. Entropy is, in this sense, a measure of the inaccessible energy. The physicist Ludwig Boltzmann proposed a statistical entropy measure $(\mathrm{H})$ :

$$
H=-K \sum_{i=1}^{N_{s}} P_{i} \log \left(P_{i}\right)
$$

Where $\mathrm{K}$ is the Boltzmann constant (only depending on the units used), and $\mathrm{P}_{\mathrm{i}}$ is the ordinary probability of an element being in any one of the $\mathrm{N}_{\mathrm{s}}$ phase space states. Shannon, particularly, reached to the same Boltzmann expression with $\mathrm{K}=1$. Kolmogorov and Sinai proposed, in 1959, to apply the Shannon entropy to dynamic systems. For such end, they used the Correlation Integral $\left(\mathrm{C}_{\mathrm{m}}(\varepsilon)\right)$ [6-10]. The computed K-S entropy $\left(\mathrm{H}_{\mathrm{KS}}\right)$ can be interpreted as a loss (or gain) of information by the system, between the m.p and $(m+1)$. $p$ instants, where $p$ is the reconstruction step:

$$
\mathrm{H}_{\mathrm{KS}}=\lim _{\varepsilon \rightarrow 0} \lim _{m \rightarrow \infty} \frac{1}{\mathrm{p}} \ln \frac{\mathrm{C}_{\mathrm{m}}(\varepsilon)}{C_{m+1}(\varepsilon)}
$$

As $m$ grows, the $K_{2}$ mean value defined as:

$$
\mathrm{K}_{2}=\frac{1}{\mathrm{p}} \ln \frac{\mathrm{C}_{\mathrm{m}}(\varepsilon)}{C_{m+1}(\varepsilon)}
$$

converges to $\mathrm{H}_{\mathrm{KS}}$. This mean value is plotted in a diagram as a function of $\mathrm{m}$, for different values of $\varepsilon$, and we look for its asymptotic value.

The main goal of the present work is to evaluate the tool: Kolmogorov-Sinai entropy (K-S) when applied to HRV time series in different powers of dynamic exercise, in order to quantify the AT in healthy individuals. The results were compared to the AT values obtained using ARIMA model.

\section{Methods}

Ten healthy male volunteers have been studied $(23 \pm$ 2.0 years) They exhibited a sedentary life style. Dynamic exercise tests (discontinuous steps) included two 
experimental protocols (undertaken two days apart), that is, progressive (EPI) and random (EPII) power levels, lasting fifteen minutes, with a rest period among them.

The RR intervals have been measured, in seconds, from each one of the following situations: at rest, in supine and seated positions; in the last position during exercise, using an electromagnetic braked cycleergometer at several power levels (W). The RR intervals were obtained using specific software to detect $R$ waves of ECG signals and the respective periods [11]. For each one of the studied powers, the ARIMA model $[3,4]$ was used to analyze the cardiac frequency responses obtained from the RR interval transformations. The K-S parameter was, then, estimated using computational programs written and compiled for the present work. Such computational programs were fed by the original RR intervals time series, dropping out the first and the final 1.5 minutes in order to assure a stability period for the studied signal.

\section{Results}

RR interval time series ( $\mathrm{N}=191)$ obtained from the ten men with both protocols, EPI and EPII, were analyzed in order to estimate the Kolmogorov-Sinai entropy for each set of data, we have plotted the values of $\mathrm{K}_{2}$ against the embedding dimension $\mathrm{m}, \mathrm{m}=2,3, \ldots, 30$ and for different values of $\varepsilon$ (Fig.1). Each RR series was reconstructed with time-delay coordinates $x(t), x(t+p)$, $\mathrm{x}(\mathrm{t}+2 \mathrm{p}), \ldots$, where the delay $\mathrm{p}$ was properly selected.

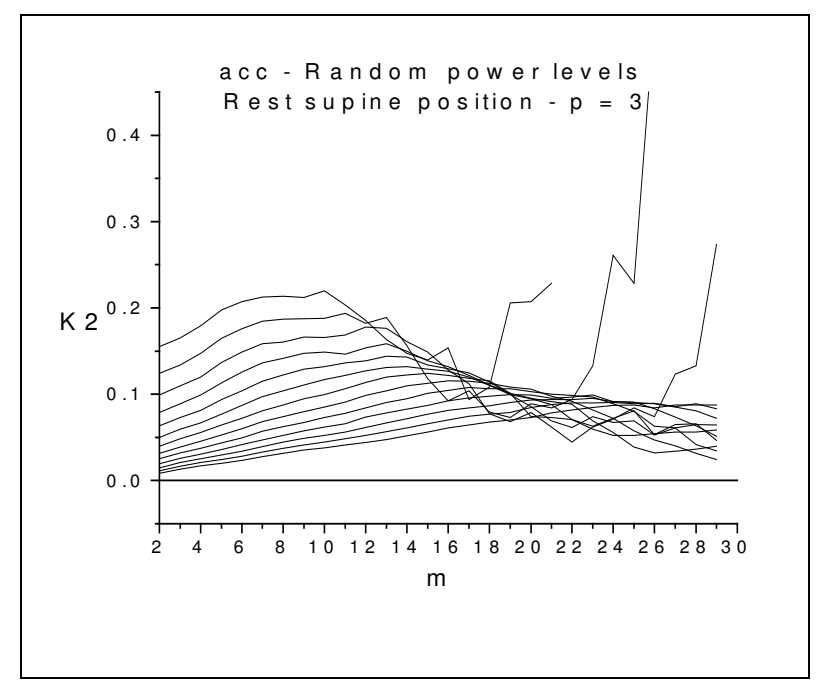

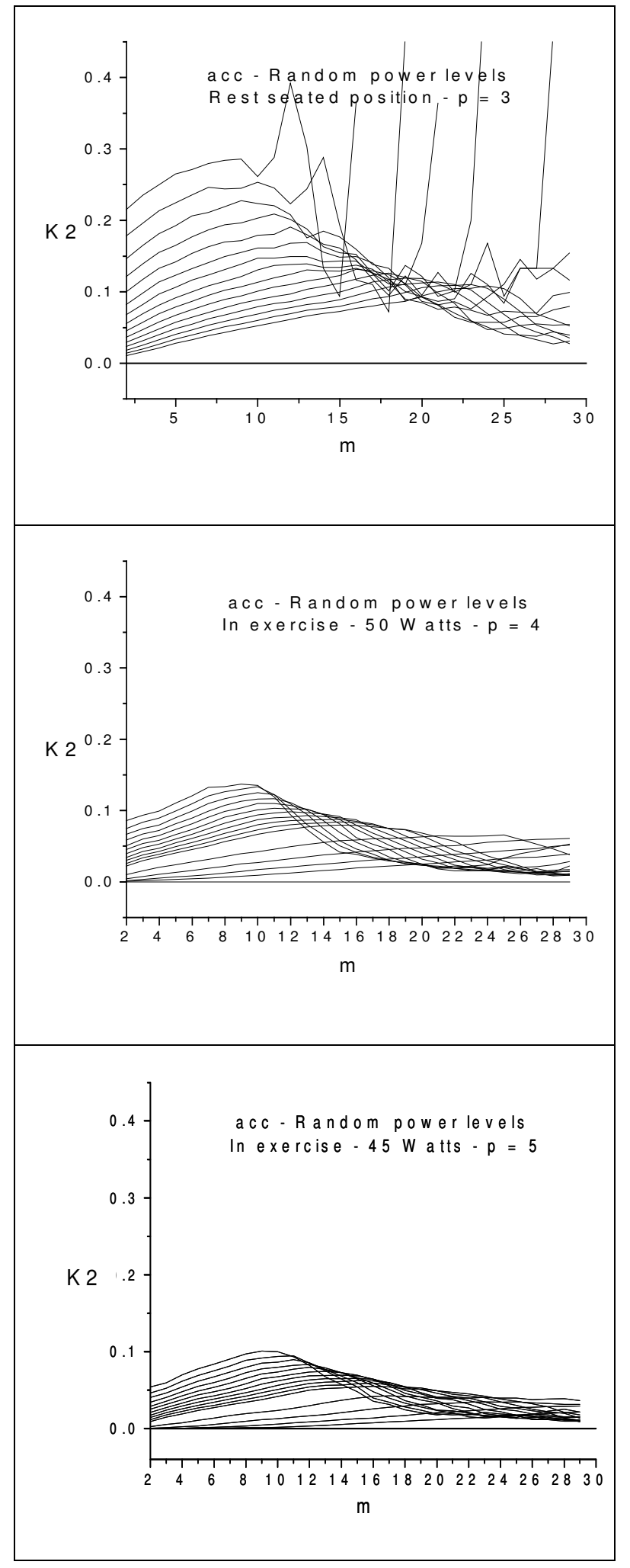



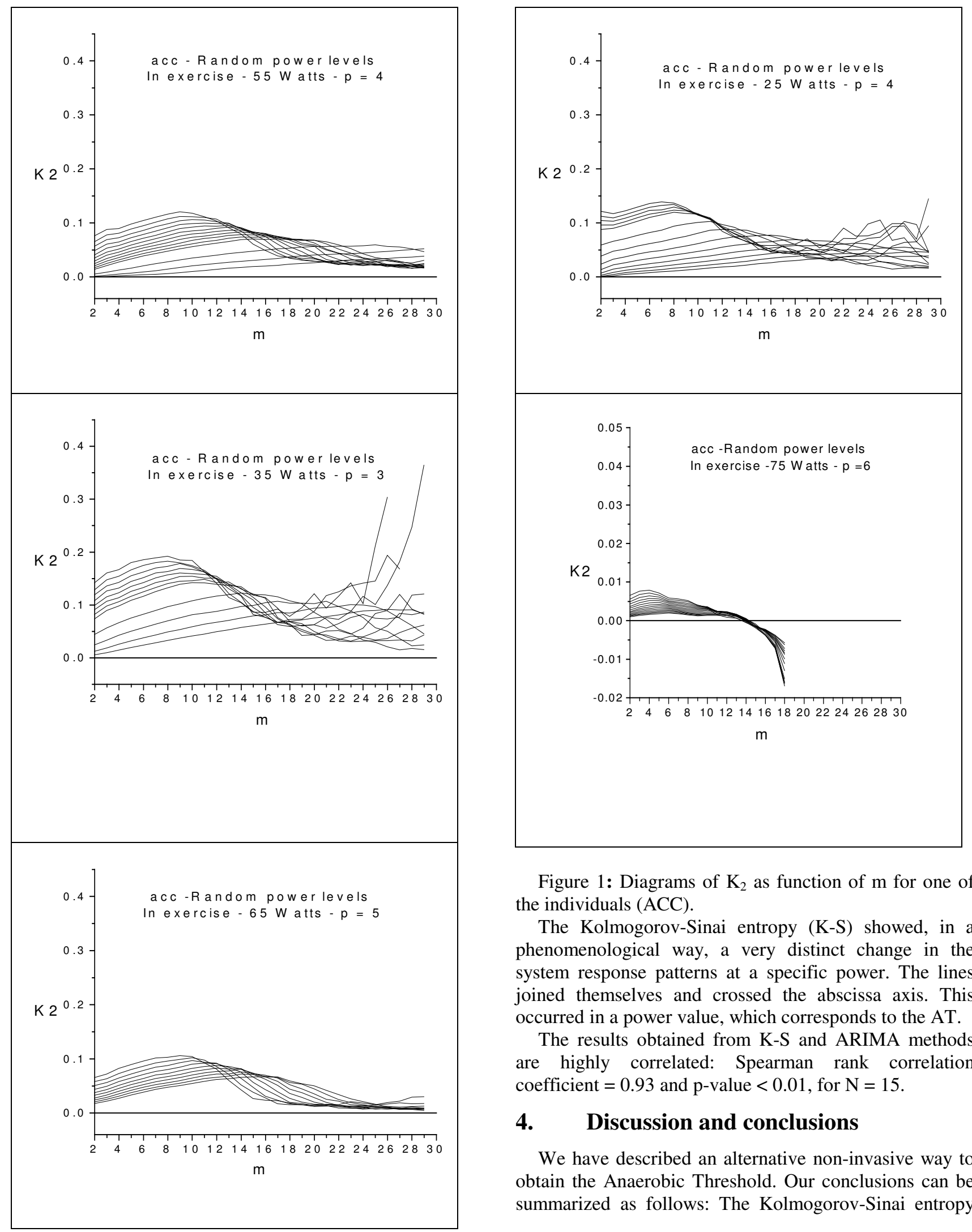

Figure 1: Diagrams of $K_{2}$ as function of $m$ for one of the individuals (ACC).

The Kolmogorov-Sinai entropy (K-S) showed, in a phenomenological way, a very distinct change in the system response patterns at a specific power. The lines joined themselves and crossed the abscissa axis. This occurred in a power value, which corresponds to the AT.

The results obtained from K-S and ARIMA methods are highly correlated: Spearman rank correlation coefficient $=0.93$ and $\mathrm{p}$-value $<0.01$, for $\mathrm{N}=15$.

\section{Discussion and conclusions}

We have described an alternative non-invasive way to obtain the Anaerobic Threshold. Our conclusions can be summarized as follows: The Kolmogorov-Sinai entropy 
exhibited, in a phenomenological way, a drastic change in the system dynamics, showing a graphic pattern completely different from the others at power values that, invariably, agreed with the AT obtained from the ARIMA model. The high linear correlation $(r=0.93)$, statistically significant at $1 \%$ level, between the ARIMA and Kolmogorov-Sinai entropy method, allows the conclusion that this last method can be used to obtain the AT, despite some problems that were expected like the data set size, different embedding dimensions and the reconstruction step size. The advantages of the Kolmogorov-Sinai entropy are:

- It involves a fast computational procedure.

- It does not require elaborate statistical analysis. This implies that a physician can perform this test without the aid of a specialized staff.

\section{Acknowledgements}

FAPESP, CNPq (nº 300528/85), FAEPA.

\section{References}

[1] Wasserman $\mathrm{K}$ et al. Principles of Exercise Testing and Interpretation, Philadelphia: Lippincott Williams and Wilkins, 1999.

[2] Gallo Jr L et al. Control of heart rate during exercise in health and disease. Brazilian Journal of Medicine and Biological Research 1995; 28: 1179-84.

[3] Box GEP, Pierce DA. Distribution of residual autocorrelations in autoregressive integrated moving average time-series models. Journal of American Statistical Association 1970; 65: 1509-26.
[4] Box GEP, Jenkins GM. Time Series Analysis: Forecasting and Control. San Francisco: Holden- Day Pub, 1976.

[5] Marães VRFS et al. The heart rate variability in dynamic exercise. Its possible role to signal anaerobic threshold. The Physiologist 2000; 43: 339.

[6] Grassberger P and Procaccia I. Measuring the strangeness of strange attractors. Physica D 1983; 9 : 189-208.

[7] Takens F. Detecting Strange Attractors in Turbulence. In: Dynamical Systems and Turbulence. Lecture Notes in Mathematics. vol. 898; 366-81, Berlin: Springer-Verlag, 1981.

[8] Schuster HG. Deterministic Chaos: an Introduction. Weinheim: Physik Verlag, 1988.

[9] Tsonis AA. Chaos: from Theory to Applications. New York: Plenum Press, 1992.

[10] Silva FMHSP. Aplicação da dinâmica não-linear no estudo da resposta dos intervalos RR do eletrocardiograma durante o exercício físico dinâmico em indivíduos sadios. $\mathrm{PhD}$. Thesis, University of São Paulo, Brazil, 2001.

[11] Silva E et al. Design of a computerized system to evaluate the cardiac function during dynamic exercise. Physics in Medicine \& Biology 1994; 33: 409.

Address for correspondence

Lourenço Gallo Junior

Division of Cardiology, Medical School of Ribeirão Preto. Av. Bandeirantes, 3900, Ribeirão Preto - SP.

CEP: 14.046-900, Brazil. E-mail: lgjunior@fmrp.usp.br 